\title{
Multiwalled Carbon Nanotubes for Amperometric Array-Based Biosensors
}

\author{
Irene Taurino • Giovanni De Micheli • Sandro Carrara
}

Published online: 27 September 2012

(C) Springer Science+Business Media, LLC 2012

\begin{abstract}
For diagnostic and therapeutic purposes an accurate determination of multiple metabolites is often required. Amperometric devices are attractive tools to quantify biological compounds due to the direct conversion of a biochemical event to a current. This review addresses recent developments in the use of multiwalled carbon nanotubes to enhance detection capability of amperometric array-based biosensors. More specifically, the principal techniques for multiwalled carbon nanotube incorporation onto microelectrode arrays are described. In these types of devices, each electrode is responsible for sensing one metabolite. The specificity is often given by an enzyme since most biomolecules are not electroactive compounds. Common strategies for the protein immobilization onto multiwalled carbon nanotubes are also presented. After the discussion of nanotube/biomolecule integration onto electrode surfaces, three results are shown. The first one regards the influence on the biodetection signal of differently oriented multiwalled carbon nanotubes. Secondly, a demonstration of enhanced biodetection parameters by using multiwalled carbon nanotubes is given. Finally, a comparative study of three enzymes used to detect the same metabolite and adsorbed onto multiwalled carbon nanotubes is also reported.
\end{abstract}

Keywords Multiwalled carbon nanotubes $\cdot$ Metabolite detection - Amperometric array-based biosensors • Enzyme immobilization • Nanostructure integration

\footnotetext{
I. Taurino $(\bowtie) \cdot$ G. De Micheli · S. Carrara

Laboratory of Integrated Systems, EPFL - École

Polytechnique Fédérale de Lausanne, 1015

Lausanne, Switzerland

e-mail: irene.taurino@epfl.ch
}

\section{Introduction}

The development of new biosensors for monitoring various metabolites in the human body is very important because they can provide an indication of potential disorders or diseases [1]. In particular, research efforts have been focused on the development of easy to use, accurate, low cost, portable, point of care sensors for efficient diagnoses and therapies. Patients' autonomy into everyday life can be improved together with their health safety by using these types of sensors, integrated into a smart electronic device.

Among different biosensors, those based on amperometric transducers confer a high sensitivity and low detection limits [2]. The current is measured in response to a variety of applied potential waveforms with voltammetric methods. The generated electrical signal relates to the concentration of an analyte [2]. These devices combine the analytical capability of electrochemical techniques with the specificity of biological recognition processes. In particular, the enzymatic activity guarantees excellent molecular specificity [3].

An additional advantage of such devices is the possibility of easy miniaturization. In such case, detection mechanisms are performed with very small solution volumes in both clinical and in home use settings. Indeed, implantable enzymatic electrochemical sensors for glucose monitoring that are commercially available are miniaturized [4]. For diagnostic purposes, it is also desirable to simultaneously monitor the concentration of two or more metabolites in human fluids such as blood, cerebrospinal fluid, subcutaneous interstitial fluid, and lymph. For instance, the levels of both glucose and glutamate in cerebrospinal fluid are important to control diseases such as meningitis [5]. 
To this aim, not only miniaturized but also fully integrated biosensor platforms for advanced healthcare is needed [6].

A continuing challenge in the fabrication of many biosensors is still the improvement of the detection performance. Nanostructured electrodes are currently used for this purpose [7]. Carbon nanotubes (CNTs) moved into the focus of research as interesting materials for the fabrication of biosensing devices with enhanced sensing parameters. The improvement of the active surface area [8], their capability to facilitate redox reactions of many compounds [9], the increase in the sensitivity from millimolar [10] to micromolar ranges [11] advocate to the use of nanotubes as key building blocks to fabricate electrodes. Enzymatic electrochemical biosensing for medical purposes is one of the most promising application of CNTs [12]. Indeed, research groups have demonstrated the nanotube capability to promote the electron transfer reactions of enzymes to the electrode. MWCNTs reduce also the fouling of enzymes once they are immobilized on these nanostructures [13]. Actually, there are two types of CNTs: single-walled CNTs (SWCNTs) that are found to be metallic, semiconductors, or small-band gap semiconductors; and multiwalled CNTs (MWCNTs) that are mostly metallic. Indeed, if a single cylinder is metallic, the whole MWCNT with cut cup displays a metallic behavior. Thus, MWCNTs are better candidates for electrochemical applications [14].

Many different types of electrode substrates and geometries have been used to fabricate electrochemical biosensors. In recent years, microelectrodes for biosensing have generated a big interest. They enable fast mass transport, high signal to noise ratio, short time to reach the steady-state signal, and ready production and need low concentrations of electroactive compounds [11]. In addition, a microelectrode array keeps the advantage to simultaneously perform a parallel detection of two or more analytes by different sensing sites [11].

This review will first give a general description of the main components characterizing biodevices based on an array of microelectrodes for multiple metabolite detection. Second, the most important methods to integrate nanostructures as MWCNTs onto a microelectrode array will be shown. Then, the common strategies for the immobilization of enzymes onto MWCNTs will be discussed. Finally, some experimental results are shown. They deal with the change of the bioelectrochemical behavior of differently oriented MWCNTs, the improved sensing parameters when MWCNTs are used for biosensing, and a comparative study of different enzymes immobilized onto MWCNTs to detect the same biocompound.

\section{Heterostructure Integration}

In the last few years, the use of miniaturized electrodes fabricated by thin-film technology has become very common in sensor research because of many advantages (e.g., smaller background current and faster time response). By using this technique, metallic films are deposited onto the insulating substrate, ensuring an excellent adhesion of the films onto the substrate. By photolithographic and etching processes, these films are structured according to the biosensor layout requirements. Minimum structure resolution ranges between 5 and $20 \mu \mathrm{m}$. Moreover, thickness of the layers can vary from nanometers to several micrometers.

The combination of microelectrode devices with biochemical compounds such as enzymes provides excellent prerequisites for the development of miniaturized biosensors to monitor in parallel many metabolites [11]. These biodevices could be composed of working electrodes that share the same counter and reference electrodes. For the fabrication of enzymatic MWCNTbased microelectrode array, working electrodes have to be nanostructured at first with MWCNTs (increase of sensitivity and decrease of detection limit). Then, enzymes have to be incorporated to make each microsurface selective for one metabolite of interest. A fixed or variable potential between reference microelectrode and working microelectrodes must be applied according to the detection molecule assigned to each working electrode surface.

\section{MWCNT Integration Methods onto Microelectrodes}

\subsection{Additive-assisted Immobilization}

Drop casting by microspotting Casting immobilization is the most widely used and simple method for fabricating MWCNT-based electrochemical sensors. With this approach, MWCNTs must be dispersed in a certain solvent under sonication after their purification and activation pretreatments. The resultant suspension must be cast onto the electrodes and allowed to dry (see Fig. 1a). To deposit MWCNTs onto microelectrodes, common volatile solvents are not suitable (chloroform [11]). Therefore, additives need to be added into solvents to assist the nanotube casting (e.g., sol-gel, nafion, chitosan, and polymers) [15]. These strategies provide 
Fig. 1 Drop casting of MWCNT-based solution by a pipette (a), microspotter with a biodevice having multiple microsensing sites (b), and setup for the electropolymerization of MWCNTs (c)

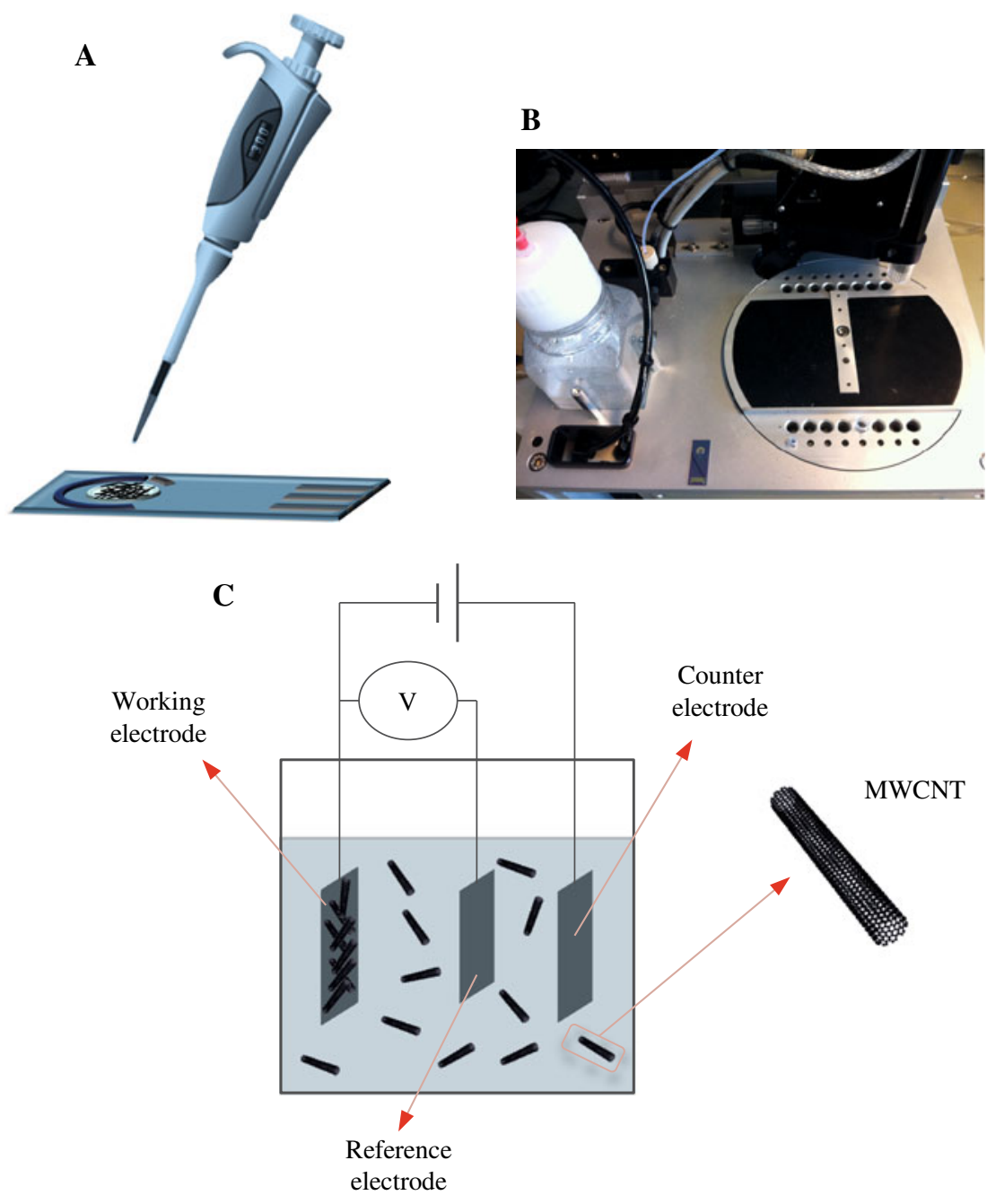

convenient approaches for an easy, robust, and noncovalent immobilization of MWCNTs onto the electrodes. However, the conductivity of MWCNTs embedded in such matrices is hindered, unavoidably affecting the sensor detection performance.

A microspotting system is used to assemble MWCNTs onto small surfaces by drop casting. A CNT suspension is transferred to the probe tip by a micropump. A probe is then moved to the desired position by using a computer-controllable micromanipulator. Finally, the CNT suspension is deposited onto the desired location [16]. Spotting CNTs needs a precise positioning of the probe due to the small solution volumes required. Lai et al. [16] employed this technique first to connect CNTs across two microelectrodes. They studied how different probes and how the probe position affect the spot size. They tested two different spotting strategies to study these outcomes of the system: injection method by a syringe pump that induces hydraulic pressure and droplet contact method (droplet in contact with the substrate). In the biosensing context, Boero et al. [17] used a MWCNT solution based on nafion. Polymeric solvent was preferred to chloroform since the latter is too volatile for the manipulation of small volumes (400 pl). A commercially available noncontact spotter was used to deposit multiple layers of MWCNT-nafion solutions onto microelectrodes of a biosensor (Fig. 1b). Solvents were allowed to dry in between two subsequent deposition steps.

Electropolymerization Another additive-assisted method to incorporate MWCNTs onto the electrode surface is the electropolymerization. In this case, nanotubes are entrapped in conductive (e.g., polypirrole [18] , polyaniline [19]) or nonconductive polymers (e.g., chitosan [20]). By applying fixed [18, 20, 21] or variable potentials $[19,21]$, monomers in solutions containing CNTs polymerize, allowing the immobilization of CNTs onto the electrode surface (Fig. 1c). Advantages related to the electropolymerization process are synthesis of the 
polymer and formation of the film in a single and rapid step (some minutes), uniformity and control of composition, molecular structure, thickness and porosity, and possibility to incorporate MWCNTs together with the biocatalyst in a polymeric film by only one step [20]. Wang et al. found that the electropolymerization rate and redox properties of the film is not highly affected by MWCNTs. They also proved that MWCNTs retained their conductive properties providing devices with high sensitivity/specificity and low detection limit [20].

\subsection{Binder-free Immobilization}

Direct self-assembly The advantage of this method is linked to the great chemical affinity between gold and thiol and to the good stability of the gold-S bond. Common to these methods is the formation of carboxylic moieties on the surface of the CNTs usually introduced by a strong acid oxidation. They occur predominantly at the more reactive sites, namely tips or defects of CNTs. Figure 2a shematically reports this type of immobilization procedure for MWCNTs. The covalent bond
SWCNTs-gold is well-established [22], especially for biodetection. Despite the fact that MWCNTs have better electron conduction properties than SWCNTs, few studies about the MWCNTs-gold binding procedures are reported in the literature. It is due to two main challenges: the higher difficulty to disaggregate and suspend MWCNTs and the difficulty to induce a preferential orientation on MWCNTs because the introduction of thiol moieties modifies the sidewalls of MWCNTs and not only their tips as in the case of SWCNTs. There are two main approaches to covalently link MWCNTs and gold: gold-S bonding and surface condensation. With the first method, carboxyl-terminated MWCNTs must be modified with a sulfanyl group. Then, substrate dipping in MWCNT solutions made with different solvent as ethanol [23], chitosan [24], and tween [24] allows us to assembly functionalized MWCNTs onto gold surfaces. The second strategy (surface condensation) consists of a dissolution of carboxyl functionalized MWCNTs into an organic solvent [25]. Moreover, gold substrates must be premodified with amino groups [25]. The reaction is obtained with 1-ethyl-3-
Fig. 2 Schematic of MWCNT self-assembly immobilization on gold (a). MWCNTs directly grown on metallic working electrodes of a biosensor by CVD (b)
A

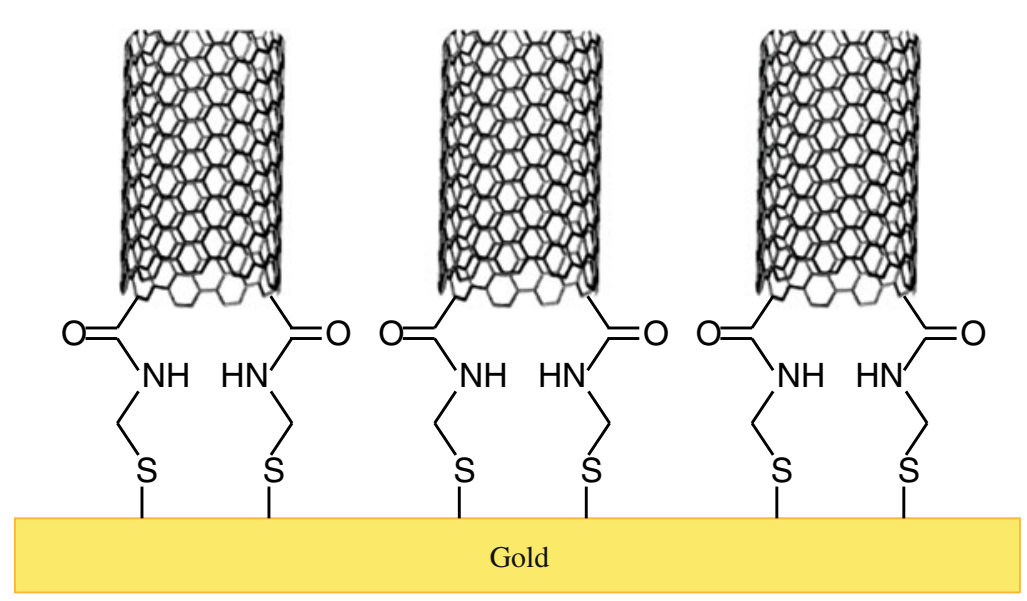

B

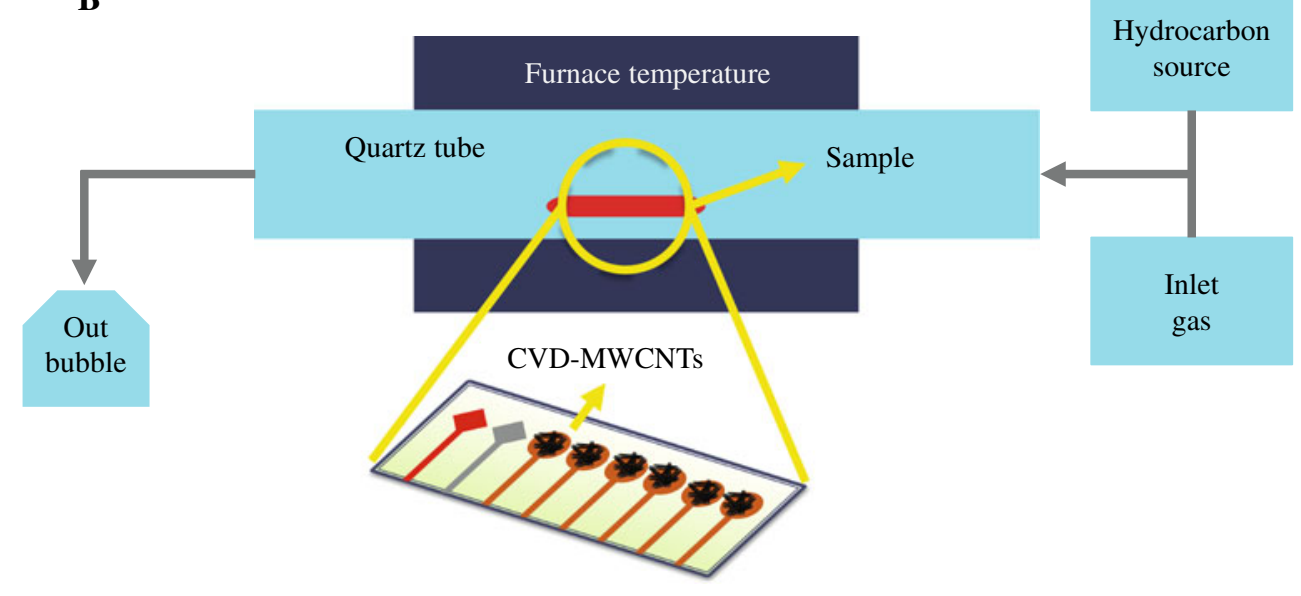


(3-dimethyl-aminopropyl) carbodiimide hydrochloride (EDC) as coupling agent. However, there are cases of mixed procedures. For instance, Kim et al. added alkanedithiols to the surfaces of MWCNTs and immobilized them on self-assembled monolayer (SAM)modified gold substrates by dipping them into DMSO solution containing MWCNTs. They found a selective adsorption of MWCNT-SH onto SAMs of alkanethiols that occurs when the dithiolates are longer than the thiolates in the SAM [26].

Chemical vapor deposition For biosensing applications, a good electrical contact between CNTs and conductive substrates is important. A possible strategy is to directly grow them by chemical vapor deposition (CVD). In CVD, a hydrocarbon gas with carbon atoms is provided for the CNT growth on metallic catalysts [27]. The biodevices must be placed in a quartz tube. A hydrocarbon gas mixture is passed over the quartz that catalytically decomposes over the metal particles (e.g., iron, nickel) previously deposited onto each sensing site (Fig. 2b). The pattern of catalytic metals can be realized onto working electrodes through either standard microfabrication techniques (e.g., UV photolithography, electron-beam evaporation, lift-off) or electrodeposition [28]. Compared to other methods of CNT production, CVD was regarded as the most promising one for industrial applications.

Unfortunately, growing CNTs on metal substrates is difficult since the elevated temperature may activate the diffusion of the catalyst material into the electrodes. This phenomenon inhibits the catalyst activity because of the coarsening and segregation during the CNT growth $[29,30]$. To overcome this problem, one possible strategy is to employ the same metal as both catalyst and electrode substrate [31] or alloys [32] containing at least one catalyst material as the electrode. If the substrate selection is constrained to specific metals, an alternative effective choice is to deposit a thin insulating layer to stop the poisoning of catalyst particles. However, insulating layers cannot provide an electrical contact in MWCNT electrode, and additional fabrication processes are required [29]. Nevertheless, some authors succeeded in growing MWCNTs directly on metal substrates (titanium thin layer [33] and platinum [28]). This works were not applied to an array of microelectrodes. Moreover, only high temperature enables the growth of MWCNTs $\left(500-1,000{ }^{\circ} \mathrm{C}\right)$. As a consequence, it is difficult to grow MWCNTs on metallic electrodes at complementary metal-oxide semiconductor (CMOS) compatible temperatures $\left(400-450{ }^{\circ} \mathrm{C}\right)$ [34]. Lowering the growth temperature has frequently proven to be ineffective because the structural quality of nanotubes usually degrades as reaction temperatures are decreased. Chen et al. [35] succeeded in growing CNTs at CMOS compatible temperature $\left(350{ }^{\circ} \mathrm{C}\right)$ but only onto insulating substrates.

Up to now, only few papers have reported this kind of MWCNT incorporation technique to fabricate enzyme biosensors. Lin et al. [36] reported a glucose biosensor based on CNT nanoelectrode ensembles made of low-site density-aligned CNTs grown on a chromium-coated silicon substrate. They used plasmaenhanced CVD and nickel nanoparticles as catalysts. An epoxy-based polymer was then spin-coated and the protruding parts of the CNTs were removed by polishing. Then, glucose oxidase was immobilized directly on the broken tips of CNTs for glucose detection. In addition, Tominaga et al. [37] studied the direct electron transfer reactions of D-fructose dehydrogenase immobilized onto MWCNTs for the D-fructose detection. In the present work, MWCNTs were synthesized by CVD using iron nanoparticles as catalysts derived from ferritin molecules and adsorbed onto a platinum plate. Due to the CNT hydrophobicity, CVD-CNTs need to be chemically and/or electrochemically treated for an easy incorporation of enzymes.

\section{MWCNT Functionalization with Bioreceptors}

Some metabolites (bilirubin, uric and ascorbic acid, and insulin) are electroactive and hence prone to a direct electrochemical detection. However, many biomolecules are not electroactive at suitable potentials (lactate and glucose). To allow the electrochemical detection of these compounds, enzymes are incorporated onto the electrodes. Studies show that nanomaterials are excellent support for the enzyme immobilization. In particular, MWCNTs are efficient to tunnel electrons to enzymes and also to improve the biomolecule electrocatalysis. The enzyme activity is for sure influenced by the use of covalent or noncovalent approaches for the protein immobilization [38].

\subsection{Covalent Bonding}

The covalent attachment of the protein to MWCNTs has been widely reported. A schematic representation of a nanotube covalently bound an enzyme is shown in Fig. 3a. Ji et al. [39] selected this type of approach to link lipase and MWCNTs. They used the common EDC and NHS coupling agents finding an improved selectivity of the lipase with respect to the native one. The enzyme was also stable after the covalent immobiliza- 
Fig. 3 Scheme of enzyme immobilization by a covalent (a) and noncovalent approach (b). Protein hydrophobic regions in blue by which the adsorption MWCNT-enzyme is realized
A

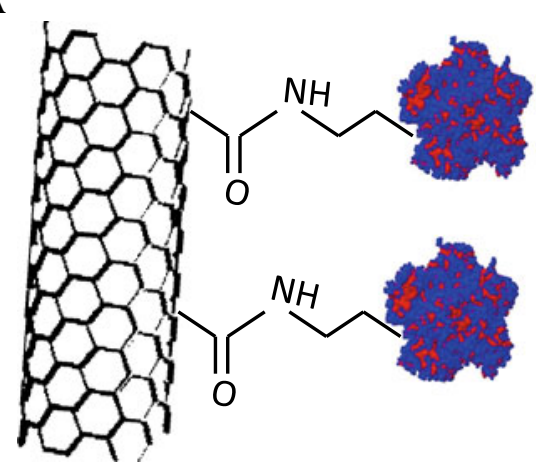

B

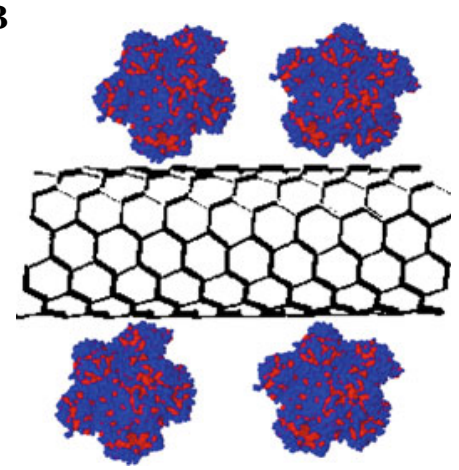

tion. Very often, extra molecules bound together with MWCNTs by hydrophobic interactions are employed for covalent bond. Basically, the added molecules provide functional groups to covalently link the enzymes. For instance, Kim et al. used 1-pyrenebutanoic acid succinimidyl ester as a cross-linker [40].

\subsection{Physical Entrapment}

Typically, the procedure to physically adsorb enzyme onto CNTs involves bathing the nanostructured electrodes in a solution of the enzyme for a certain time. Physical adsorption studies have proved the natural affinity between enzymes and MWCNTs [41, 42]. Adsorbed enzymes denature less upon immobilization and the intrinsic electronic structure and properties of CNTs are preserved [41]. With this approach, both the hydrophobic and electrostatic interactions play an important role (Fig. 3b) [43]. Moreover, in the presence of MWCNTs with functional groups, the hydrogen bonding interactions can support the adhesion of enzyme and nanotube [11]. Some studies report the enzyme adsorption in polymer-MWCNT composites. For example, Lee et al. [44] utilized a layer of sulfonated polyaniline network on MWCNTs and adsorbed glucose oxidase. The modified sensor showed fast electron transfer for the enzyme, wide linear range, and high sensitivity for the determination of glucose as well as a good reproducibility.

\section{Electrochemical Behavior of Differently Oriented MWCNTs}

The electrochemical properties of MWCNTs are strongly dependent on pretreatments (e.g., chemical, electrochemical) as well as densities and orientations (e.g., randomly oriented or well-organized nanostructures). In order to gain fundamental insight about the bioelectrosensing behavior of MWCNTs, we directly grew them onto nonconductive silicon wafers via CVD (SEM images in Fig. 4) and evaluated the sensing performance by cyclic voltammetry. We also studied the nanotube wettability since it is important to have hydrophilic nanostructured electrode for an easy incorporation of enzymes. We considered the electrochemistry of the hydrogen peroxide because it is a product of reactions catalyzed by many enzymes, like oxidases and peroxidases. It can be oxidized at the electrode surface at $+650 \mathrm{mV}$ [45] according to the following reaction Eq. 1:

$\mathrm{H}_{2} \mathrm{O}_{2} \stackrel{+650 \mathrm{mV}}{\longrightarrow} 2 \mathrm{H}^{+}+\mathrm{O}_{2}+2 e^{-}$

The wettability of MWCNTs with only exposed tips (Fig. 3c) and with also exposed walls (Fig. 3a,b,d) before and after an acid treatment revealed that treatment acts on the nanotube walls creating structural defects. Indeed, vertically packed MWCNTs (Fig. 3c) maintain their characteristic hydrophobicity (contact angle close to $107^{\circ}$ ), while nanotubes randomly oriented with respect to the substrate become hydrophilic after the acid treatment, showing a contact angle of close to $24^{\circ}$. Interestingly, the introduction of wall defects improves the detection of hydrogen peroxide. Indeed, sensitivity goes from $28.8 \pm 0.1 \mu \mathrm{A} /\left(\mathrm{mM} \mathrm{cm}^{2}\right)$ to $59.2 \pm 0.4 \mu \mathrm{A} /\left(\mathrm{mM} \mathrm{cm}^{2}\right)$ when randomly oriented nanotubes are treated [46, 47].

It is known that the electron transfer on CNTs takes place at their tips [48]. The role of CNT walls is still under investigation [49]. So, we evaluated the electrochemistry of electrodes nanostructured with four differently oriented and acid-treated MWCNTs [50]: pillars of double-bedded MWCNTs (Fig. 4a), islands of horizontally/randomly oriented MWCNTs (Fig. 4b), densely packed vertical MWCNTs (Fig. 4c), and vertically aligned MWCNTs with oriented tilted tips (Fig. 4d) [50]. In this study, MWCNTs with tilted tips showed the best electrochemical behavior towards the 
Fig. 4 FE-SEM images of differently oriented MWCNTs grown on Si wafer: pillars of MWCNTs (a), islands of randomly/horizontally oriented MWCNTs (b), vertical MWCNTs (c), and MWCNTs with tilted tips (d)
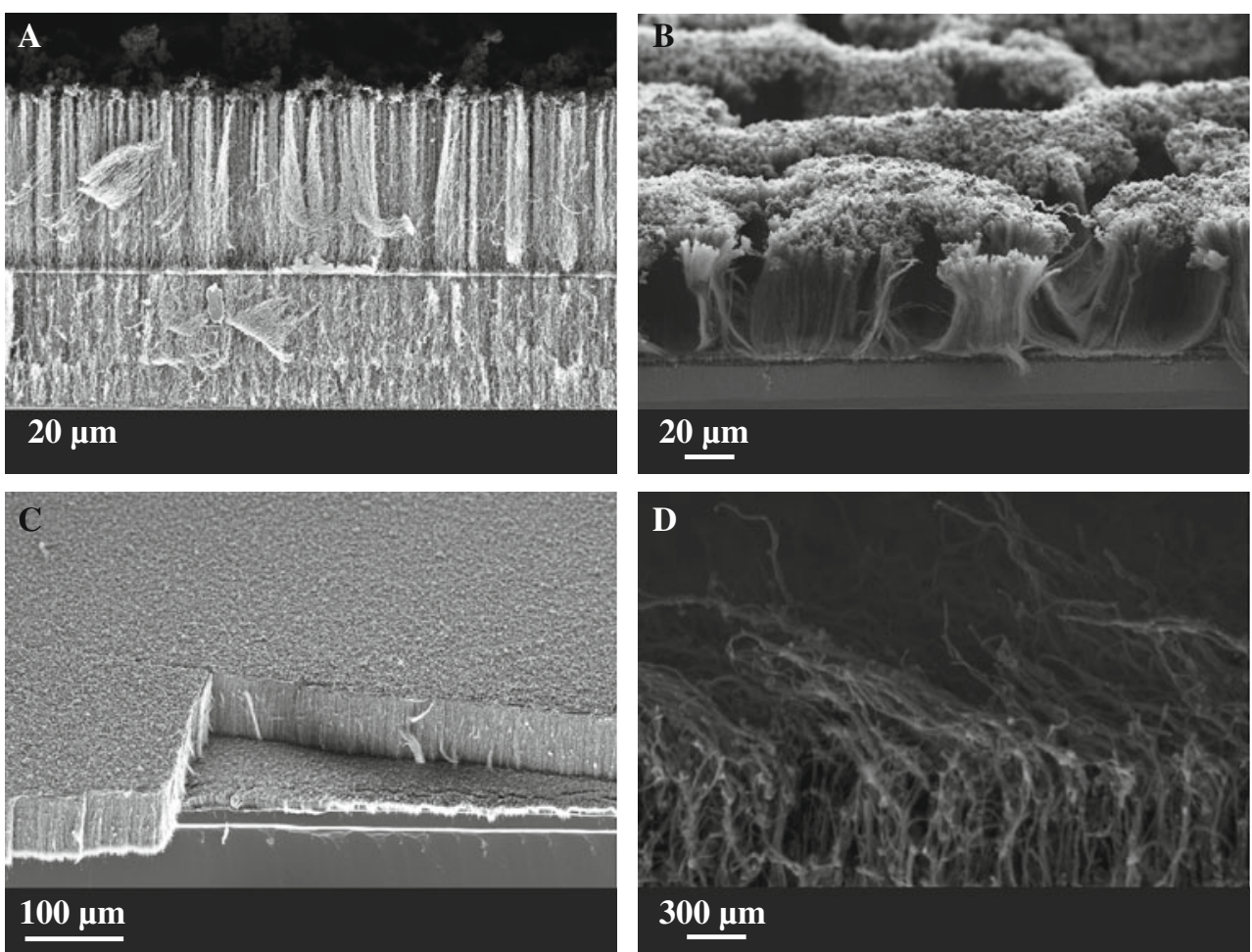

detection of hydrogen peroxide (see Table 1). This result is explained by the electroactive contribution of both edge-plane-graphite-like open ends and defects sites. Moreover, the last nanostructure offers the lowest oxidation potential of hydrogen peroxide (see Table 1). Considering also the large increase in wettability (close to $22^{\circ}$ ) after the acid treatment, we can argue that MWCNTs with tilted tips are the most suitable for an efficient immobilization of enzymes to develop amperometric biosensors.

We realized this investigation since the chance to increase the reactivity of CNTs by varying both pretreatments and the CNT tilt with respect to the electrode substrate offers the opportunity to fabricate biodevices with promising target-selective electroanalytical performance. Moreover, the CNT nanometer size favors the fabrication of miniaturized transducers.

Table 1 Electrochemical sensing parameters computed using multiple oriented MWCNTs for $\mathrm{H}_{2} \mathrm{O}_{2}$ detection

\begin{tabular}{lllc}
\hline MWCNTs & $\begin{array}{l}\text { Oxidative } \\
\text { potential }(\mathrm{mV})\end{array}$ & $\begin{array}{l}\text { Sensitivity } \\
\left(\mu \mathrm{A} /\left(\mathrm{mM} \mathrm{cm}^{2}\right)\right)\end{array}$ & $\begin{array}{c}\text { Detection } \\
\text { limit }(\mu \mathrm{M})\end{array}$ \\
\hline Vertical & 718 & $10.1 \pm 0.1$ & $252 \pm 1$ \\
Tilted tips & 570 & $16.4 \pm 0.1$ & $23.99 \pm 0.01$ \\
Horizontal & 633 & $1.81 \pm 0.04$ & $1534 \pm 25$ \\
Pillars & 593 & $3.3 \pm 0.1$ & $1623 \pm 28$ \\
\hline
\end{tabular}

\section{Enhanced Sensing Parameters with MWCNTs}

Among different biomolecules of considerable significance in clinical diagnosis, some biocompounds are electroactive and so measurable by electroanalytical methods without the use of bioreceptors. The physiological levels of biomolecules are often very low, ranging from micro [51] (bilirubin) to nano [52] and picomolar [53] concentrations (insulin). As a consequence, it is difficult to determine them at bare electrode. To overcome this problem, nanomaterials as MWCNTs are used to modify bare electrode. Below, there is an example of the improved sensing performance by employing MWCNTs to detect bilirubin, the principal component of red blood cells. Higher and lower concentrations of this biomolecule are signs of serious health problems that require medical attention. For instance, jaundice, caused by high BR levels in the blood, is associated with gallbladder and liver diseases (e.g., cirrhosis, hepatitis), blood infection, transfusion reaction, or hemolytic disease of the newborn (cell destruction) [54]. On the other hand, low level of $\mathrm{BR}$ is associated with anemias and coronary artery diseases [54].

Superior sensing performance of MWCNT-based electrodes to detect total bilirubin We prepared nanostructured electrodes by drop casting MWCNTs dissolved in chloroform onto graphite screen printed electrodes. 
We used various bilirubin concentrations ranging from 0 to $150 \mu \mathrm{M}$ that corresponds to the bilirubin physiopathological range. Cyclic voltammograms were found to be similar to those reported in the literature [55] (Fig. 5). Higher peaks were obtained using the MWCNTs-based sensor (see Fig. 5). Nanostructured electrodes have shown the better sensing performance. For bare electrodes, we obtained values of sensitivity and detection limit equal to $5.5 \pm 0.9 \mu \mathrm{A} /\left(\mathrm{mM} \mathrm{cm}^{2}\right)$ and $57.0 \pm 9.0 \mu \mathrm{M}$, respectively. On the other hand, sensitivity and detection limit with nanostructured electrodes were $83.4 \pm 1.9 \mu \mathrm{A} /\left(\mathrm{mM} \mathrm{cm}^{2}\right)$ and $8.0 \pm 0.2 \mu \mathrm{M}$, respectively.

Measurements in presence of albumin in case of critical levels Since bilirubin is almost insoluble in aqueous solution, it is found in human fluids in combination
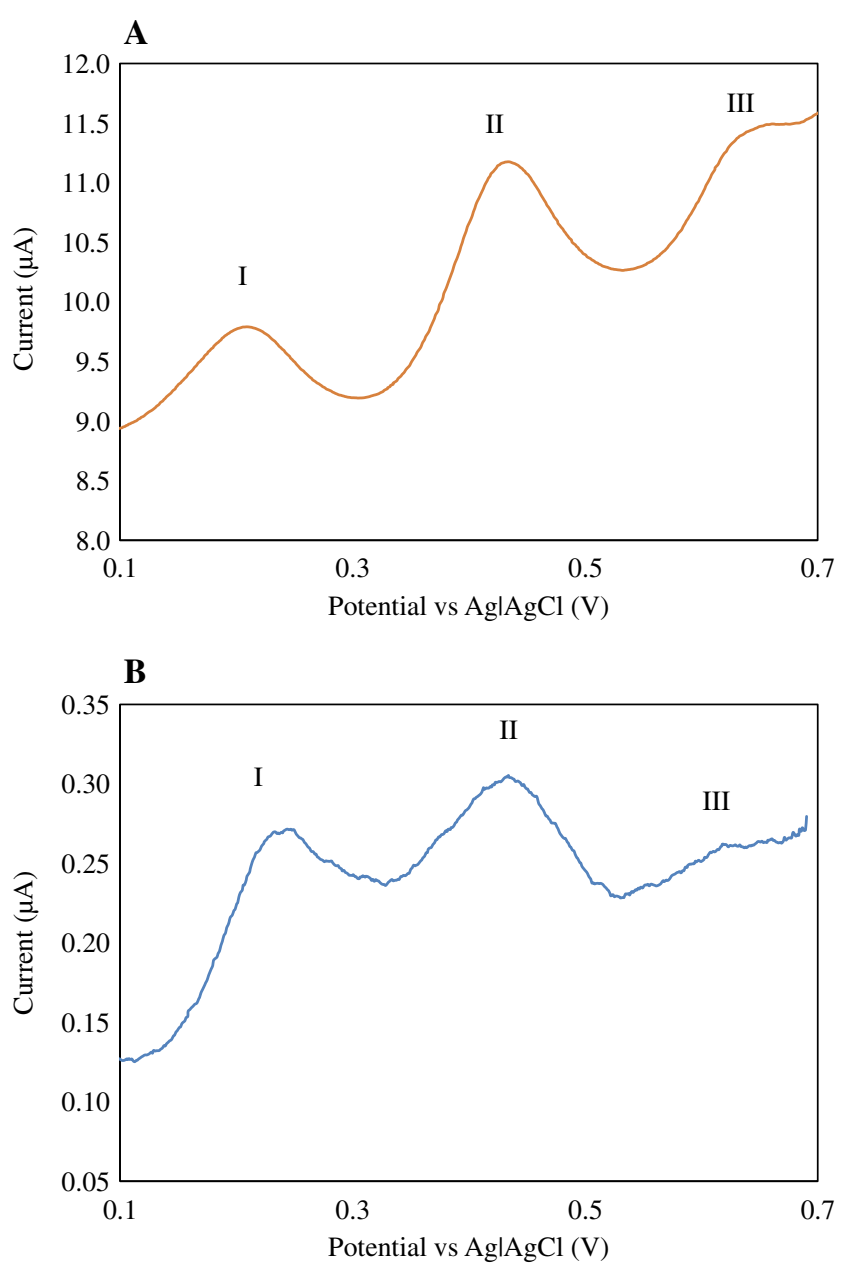

Fig. 5 Three current peaks at bilirubin concentration equal to $150 \mu \mathrm{M}$ by using MWCNT-based (a) and bare (b) sensors. They relate with the conversion bilirubin-biliverdin (peak $I$ ), biliverdin-purpurine (peak II), and purpurine-choletelin (peak III) with albumin forming with this protein a soluble complex. Albumin inhibits the electrochemical reactivity of bilirubin, making difficult the detection. We measured bilirubin in the critical range for newborns in case of jaundice (from 200 up to $400 \mu \mathrm{M}$ ) in the presence of an albumin concentration we can find in physiological conditions $(30 \mathrm{mg} / \mathrm{ml})$. Only in presence of MWCNTs that we were able to detect bilirubin. Sensitivity was $4.8 \pm 0.8 \mu \mathrm{A} /\left(\mathrm{mM} \mathrm{cm}^{2}\right)$ and detection limit was equal to $161 \pm 27 \mu \mathrm{M}$. Detection limit was below the minimum critical BR value for newborns. Therefore, our nanostructured sensor could be used to detect this metabolite in babies affected by both acute or chronic neonatal jaundice.

\section{Engineered Enzymes for MWCNT-based Electrodes}

Many 0efforts have been made to fabricate effective and more stable biosensors for accurate medical diagnosis. One issue is the difficulty to find a natural enzyme with all the features required to construct an ideal biosensor. Among different strategies, protein engineering is helping researchers to produce tailordesigned biorecognition molecules for their integration into biosensing platforms. The careful selection of the location and type of mutations (genetically created) gives rise to enzymes with enhanced or particular properties, e.g., higher affinity towards specific analytes, higher stability, higher electron transfer rates, and residues able to provide an oriented or more stable immobilization [56, 57]. Up to now, histidine-modified enzymes have been used for a covalent attachment to CNTs [56]. We firstly compared the behavior of three L-lactate oxidases from Aerococcus viridans (Lox) once immobilized by physical adsorption onto MWCNTs [58]: two engineered enzymes, Lox with N-terminal His-tag (NLox) and Lox without N-terminal His-Tag in a wild form (wtLox) and a commercial Lox (cLox). The reaction catalyzed by Lox is (Eq. 2)

$\mathrm{L}$ - lactate $+\mathrm{O}_{2} \stackrel{\text { Lox }}{\rightarrow}$ Piruvate $+\mathrm{H}_{2} \mathrm{O}_{2}$.

Then, the hydrogen peroxide can be oxidized at the electrode surface according to Eq. 1.

Using wtLox, the electrochemical response was obtained only with the $50 \%$ of the samples prepared with the same quantity of adsorbed enzyme. The relative sensitivity was equal to $18.9 \pm 3.9 \mu \mathrm{A} /\left(\mathrm{mM} \mathrm{cm}^{2}\right)$ and the detection limit was $110 \pm 12 \mu \mathrm{M}$. All the electrodes prepared with NLox and cLox showed an electrochemical response. With NLox, sensitivity resulted $\left.35.6 \pm 3.1 \mu \mathrm{A} /(\mathrm{mM} \mathrm{cm})^{2}\right)$ and detection limit 
Table 2 Electrochemical sensing parameters computed with NLox-MWCNT-based sensors during the first 50 days after the enzyme deposition

\begin{tabular}{lcc}
\hline Day & $\begin{array}{l}\text { Sensitivity } \\
\left(\mu \mathrm{A} /\left(\mathrm{mM} \mathrm{cm}^{2}\right)\right)\end{array}$ & $\begin{array}{c}\text { Detection } \\
\text { limit }(\mu \mathrm{M})\end{array}$ \\
\hline I & $35.6 \pm 3.1$ & $29.7 \pm 2.9$ \\
VIII & $38.5 \pm 3.3$ & $57.7 \pm 11.3$ \\
XVIII & $28.8 \pm 3.7$ & $47.7 \pm 8.8$ \\
XLVIII & $15.3 \pm 0.9$ & $72.4 \pm 1.2$ \\
LVIII & $7.2 \pm 1.9$ & $146.1 \pm 21.1$ \\
\hline
\end{tabular}

was $30 \pm 3 \mu \mathrm{M}$. Using cLox, sensitivity was equal to $25.6 \pm 3.4 \mu \mathrm{A} /\left(\mathrm{mM} \mathrm{cm}^{2}\right)$, and detection limit was determined to be $58 \pm 12 \mu \mathrm{M}$. The linear range was $0-0.8 \mathrm{mM}$ for wtLox and $0-1 \mathrm{mM}$ when NLox and cLox were used. It was possible to study the time stability of the NLox-based sensors only. Sensitivities and detection limit computation shows that the enzyme remains active up to 50 days after its deposition (Table 2).

\section{Conclusions}

This article summarizes recent advances in using MWCNTs for enzymatic and nonenzymatic amperometric biosensors based on multiple microelectrodes. Beyond the MWCNT excellent electronic properties, these nanomaterials increase the surface active area facilitating also the electron transfer between electrodes and proteins when incorporated onto electrochemical working electrodes. Moreover, the nanotube nanosize offer the opportunity to be incorporated onto microelectrode surfaces. Due to the well-known nanotube poor solubility, the MWCNT immobilization strategies onto small surfaces is often realized by polymeric binders via microspotting or electropolymerization. However, these integration approaches hide the excellent properties of MWCNTs due to the presence of additional materials. On the other hand, binderfree techniques give the advantage to favor the direct contact of nanotube and metallic surface, but the present techniques are still under investigation due to the needs of rigorous conditions to be realized. When a biomolecule is not electroactive at a practical redox potential, an enzyme needs to be incorporated onto the electrode. In this context a key issue is the selection of the type of enzyme (engineered or not) and the relative immobilization approach. By using MWCNTnanostructured electrodes, enzymes can be covalently or not covalently immobilized.

The necessity to simultaneously measure multiple metabolites is of increased interest in clinical field. This article proves the possibility to produce ad- vanced biosensors by using fully integrated MWCNTmicroelectrode array. Future work will address the optimization of MWCNT-microelectrode integration as well as the improvement of the bioreceptor incorporation to fabricate biodevices with enhanced performance for a wide range of clinical applications.

Acknowledgements The authors would like to thank Alberto Tagliaferro e Mauro Giorcelli for the MWCNTs fabrications and Linda Thöny-Meyer for providing NLox and wtLox. The research is supported by the i-IronIC project. The i-IronIC project is financed with a grant from the Swiss Nano-Tera.ch initiative and evaluated by the Swiss National Science Foundation.

\section{References}

1. Pejcic, B., De Marco, R., Parkinson, G. (2006) The role of biosensors in the detection of emerging infectious diseases. Analyst, 131(10), 1079-1090.

2. Lojou, A., \& Bianco, P. (2006) Application of the electrochemical concepts and techniques to amperometric biosensor devices. Journal of Electroceramics, 16, 79-91.

3. Ronkainen, N.J., Halsall, H.B., Heineman, W.R. (2010) Electrochemical biosensors. Chemical Society reviews, 39(5), $1747-1763$.

4. Wang, J. (2001) Glucose biosensors: 40 years of advances and challenges. Electroanalysis, 13(12), 983.

5. Spranger, M., Krempien, S., Schwab, S., Maiwald, M., Bruno, K., Hacke, W. (1996) Excess glutamate in the cerebrospinal fluid in bacterial meningitis. Journal of the Neurological Sciences, 143(12), 126-131.

6. Carrara, S., Ghoreishizadeh, S.S., Olivo, J., Taurino, I., BajRossi, C., Cavallini, A., de Beeck, M.O., Dehollain, C., Burleson, W., Moussy, F.G., Guiseppi-Elie, A., De Micheli, G. (2012) Fully integrated biochip platforms for advanced healthcare. Sensors, Accepted Manustript.

7. Ferrari, M. (2005) Cancer nanotechnology: opportunities and challenges. Nature Reviews Cancer, 5(3), 161-171.

8. Valentini, F., Amine, A., Orlanducci, S., Terranova, M.L., Palleschi, G. (2003) Carbon nanotube purification: preparation and characterization of carbon nanotube paste electrodes. Analytical chemistry, 75(20), 5413-5421.

9. Liu, J., Chou, A., Rahmat, W., Paddon-Row, M.N., Gooding, J.J. (2005) Achieving direct electrical connection to glucose oxidase using aligned single walled carbon nanotube arrays. Electroanalysis, 17(1), 38-46.

10. Joseph, S., Rusling, J.F., Lvov, Y.M., Friedberg, T., Fuhr, Y.M. (2003) An amperometric biosensor with human CYP3A4 as a novel drug screening tool. Biochemical pharmacology, 65(11), 1817-1826.

11. Carrara, S., Cavallini, A., Erokhin, V., De Micheli, G. (2011) Multi-panel drugs detection in human serum for personalized therapy. Biosensors and Bioelectronics, 26(9), 3914-3919.

12. Katz, E., \& Willner, I. (2004) Biomolecule-functionalized carbon nanotubes: applications in nanobioelectronics. ChemPhysChem, 5(8), 1084-1104.

13. Gong, K., Yan, Y., Zhang, M., Su, L., Xiong, S., Mao, L. (2005) Electrochemistry and electroanalytical applications of carbon nanotubes: a review. Analytical sciences, 21(12), 13831393.

14. Ajayan, P.M. (1999) Nanotubes from carbon. Chemical Reviews, 99(7), 1787-1800. 
15. Hu, C., \& Hu, S. (2009) Carbon nanotube-based electrochemical sensors: principles and applications in biomedical systems. Journal of Sensors doi:10.1155/2009/187615 .

16. Lai, K.W.C., Fung, C.K.M., Wong, V.T.S., Sin, M.L.Y., Li, W.J., Kwong, C.P. (2006) Development of an automated microspotting system for rapid dielectrophoretic fabrication of bundled carbon nanotube sensors. IEEE Transactions on Automation Science and Engineering, 3(3), 218-227.

17. Boero, C., Carrara, S., De Micheli, G. (2011) New technologies for nanobiosensing and their applications to real-time monitoring. In Biomedical Circuits and Systems Conference (BioCAS), 2011 IEEE, (pp. 357-360).

18. Hojati-Talemi, P., Simon, G.P. (2010) Electropolymerization of polypyrrole/carbon nanotube nanocomposite films over an electrically nonconductive membrane. The Journal of Physical Chemistry C 14 (33), 13962-13966.

19. Wang, Z., Yuan, J., Li, M., Han, D., Zhang, Y., Shen, Y., Niu, L., Ivaska, A. (2007) Electropolymerization and catalysis of well-dispersed polyaniline/carbon nanotube/gold composite. Journal of Electroanalytical Chemistry, 599(1), 121-126.

20. Wang, J., \& Musameh, M. (2005) Carbon-nanotubes doped polypyrrole glucose biosensor. Analytica Chimica Acta, 539(1), 209-213.

21. Vidal, J.C., Garcia, E., Castillo, J.R. (1998) Electropolymerization of pyrrole and immobilization of glucose oxidase in a flow system: influence of the operating conditions on analytical performance. Biosensors and Bioelectronics, 13(3), 371-382.

22. Yu, X., Chattopadhyay, D., Galeska, I., Papadimitrakopoulos, F., Rusling, J.F. (2003) Peroxidase activity of enzymes bound to the ends of single-wall carbon nanotube forest electrodes. Electrochemistry Communications, 5(5), 408-411.

23. Minati, L., Speranza, G., Torrengo, S., Toniutti, L., Migliaresi, C., Maniglio, D., Ferrari, M., Chiasera, A. (2010) Characterization of thiol-functionalized carbon nanotubes on gold surfaces. Surface Science, 604(17-18), 1414-1419.

24. Julio, C., Gardona, Z., Cao, R., Suarez, M. (2012) Vertical self-assembly of modified multiwalled carbon nanotubes on gold surfaces induced by chitosan and tween. Chemical Communications, 48, 1910-1912.

25. Juan, X.J., Gang, W., Zhang, X.H.X.I.A.Q., Hong Yuan, C. (2005) 3rd generation horseradish peroxidase biosensor based on self-assembling carbon nanotubes to gold electrode surface. Chinese Chemical Letters, 16(4), 523-526.

26. Kim, H.R., Park, S., Jung, C., Kim, J., Rhee, C.K., Hyun, M.S. (2010) Selective adsorption of dithiolate-modified multi-wall carbon nanotubes onto alkanethiol self-assembled monolayers on $\mathrm{Au}$ (111). Chemical Communications, 46(35), 65846586.

27. Cassell, A.M., Raymakers, J.A., Kong, J., Dai, H. (1999) Large scale CVD synthesis of single-walled carbon nanotubes. The Journal of Physical Chemistry B, 103(31), 64846492.

28. Zanganeh, S., Torabi, M., Kajbafvala, A., Zanganeh, N., Bayati, M.R., Molaei, R., Zargar, H.R., Sadrnezhaad, S.K. (2010) CVD fabrication of carbon nanotubes on electrodeposited flower-like fe nanostructures. Journal of Alloys and Compounds, 507(2), 494-497.

29. Nessim, G.D., Acquaviva, D., Seita, M., O’Brien, K.P., Thompson, C.V. (2010) The critical role of the underlayer material and thickness in growing vertically aligned carbon nanotubes and nanofibers on metallic substrates by chemical vapor deposition. Advanced Functional Materials, 20(8), 1306-1312.

30. Bayer, B.C., Hofmann, S., Castellarin-Cudia, C., Blume, R., Baehtz, C., Esconjauregui, S., Wirth, C.T., Oliver, R.A.,
Ducati, C., Knop-Gericke, A., et al. (2011) Support-catalystgas interactions during carbon nanotube growth on metallic ta films. The Journal of Physical Chemistry C 115 (11), 43594369.

31. Martin-Fernandez, I., Gabriel, G., Rius, G., Villa, R., PerezMurano, F., Lora-Tamayo, E., Godignon, P. (2009) Vertically aligned multi-walled carbon nanotube growth on platinum electrodes for bio-impedance applications. Microelectronic Engineering, 86(4-6), 806-808.

32. Talapatra, S., Kar, S., Pal, K.S., Vajtai, R., Ci, L., Victor, P., Shaijumon, M.M., Kaur, S., Nalamasu, O., Ajayan, M.P. (2006) Direct growth of aligned carbon nanotubes on bulk metals. Nat Nano, 1(2), 112-116.

33. Mendoza, E., Henley, S.J., Poa, C.H.P., Chen, G.Y., Giusca, C.E., Adikaari, A.A.D.T., Carey, J.D., Silva, S.R.P. (2005) Large area growth of carbon nanotube arrays for sensing platforms. Sensors and Actuators B: Chemical, 109, 75-80.

34. Matthews, K.D., Lemaitre, M.G., Kim, T., Chen, H., Shim, M., Zuo, J.M. (2006) Growth modes of carbon nanotubes on metal substrates. Journal of Applied Physics, 100, 044309.

35. Chen, G.Y., Jensen, B., Stolojan, V., Silva, S.R.P. (2011) Growth of carbon nanotubes at temperatures compatible with integrated circuit technologies. Carbon, 49(1), 280-285.

36. Lin, Y., Lu, F., Tu, Y., Ren, Z. (2004) Glucose biosensors based on carbon nanotube nanoelectrode ensembles. Nano Letters, 4(2), 191-195.

37. Tominaga, M., Nomura, S., Taniguchi, I. (2009) D-fructose detection based on the direct heterogeneous electron transfer reaction of fructose dehydrogenase adsorbed onto multiwalled carbon nanotubes synthesized on platinum electrode. Biosensors and Bioelectronics, 24(5), 1184-1188.

38. Mateo, C., Palomo, J.M., Fernandez-Lorente, G., Guisan, J.M., Fernandez-Lafuente, R. (2007) Improvement of enzyme activity, stability and selectivity via immobilization techniques. Enzyme and Microbial Technology, 40(6), 14511463.

39. Ji, P., Tan, H., Xu, X., Feng, W. (2010) Lipase covalently attached to multiwalled carbon nanotubes as an efficient catalyst in organic solvent. AIChE Journal, 56(11), 3005-3011.

40. Kim, B.J., Kang, B.K., Bahk, Y.Y., Yoo, K.H., Lim, K.J. (2009) Immobilization of horseradish peroxidase on multiwalled carbon nanotubes and its enzymatic stability. Current Applied Physics, 9(4), e263-e265.

41. Cang-Rong, J.T., \& Pastorin, G. (2009) The influence of carbon nanotubes on enzyme activity and structure: investigation of different immobilization procedures through enzyme kinetics and circular dichroism studies. Nanotechnology, 20, 255102.

42. Baj-Rossi, C., De Micheli, G., Carrara, S. (2012) Electrochemical detection of anti-breast-cancer agents in human serum by cytochrome p450-coated carbon nanotubes. Sensors, 12(5), 6520-6537.

43. Feng, W., \& Ji, P. (2011) Enzymes immobilized on carbon nanotubes. Biotechnology Advances 29, (6), 889-895.

44. Lee, K.P., Komathi, S., Nam, N.J., Gopalan, A.I. (2010) Sulfonated polyaniline network grafted multi-wall carbon nanotubes for enzyme immobilization, direct electrochemistry and biosensing of glucose. Microchemical Journal, 95(1), 74-79.

45. Huang, J., Song, Z., Li, J., Yang, Y., Shi, H., Wu, B., Anzai, J., Osa, T., Chen, Q. (2007) A highly-sensitive L-lactate biosensor based on sol-gel film combined with multi-walled carbon nanotubes (MWCNTs) modified electrode. Materials Science and Engineering: C, 27(1), 29-34.

46. Taurino, I., Carrara, S., Giorcelli, M., Tagliaferro, A., De Micheli, G. (2011) Comparing the enhanced sensing 
interfaces of differently oriented carbon nanotubes onto silicon for bio-chip applications. In 4th IEEE International Workshop on Advances in Sensors and Interfaces (IWASI), 2011, (pp. 90-93).

47. Taurino, I., Carrara, S., Giorcelli, M., Tagliaferro, A., De Micheli, G. (2012) Carbon nanotubes with different orientations for electrochemical biodevices. IEEE Sensors Journal, (99), 1

48. Banks, C.E., Davies, T.J., Wildgoose, G.G., Compton, R.G. (2005) Electrocatalysis at graphite and carbon nanotube modified electrodes: edge-plane sites and tube ends are the reactive sites. ChemInform, 36(18), 829-841.

49. Gong, K., Chakrabarti, S., Dai, L. (2008) Electrochemistry at carbon nanotube electrodes: is the nanotube tip more active than the sidewall? Angewandte Chemie International Edition, 47(29), 5446-5450.

50. Taurino, I., Carrara, S., Giorcelli, M., Tagliaferro, A., De Micheli, G. (2011) Comparing sensitivities of differently oriented multi-walled carbon nanotubes integrated on silicon wafer for electrochemical biosensors. Sensors and Actuators B: Chemical B 160, 327-333.

51. Shoham, B., Migron, Y., Riklin, A., Willner, I., Tartakovsky, B. (1995) A bilirubin biosensor based on a multilayer network enzyme electrode. Biosensors and Bioelectronics, 10(34), 341-352.
52. Zhang, M., Mullens, C., Gorski, W. (2005) Insulin oxidation and determination at carbon electrodes. Analytical chemistry, 77(19), 6396-6401.

53. Salimi, A., Mohamadi, L., Hallaj, R., Soltanian, S. (2009) Electrooxidation of insulin at silicon carbide nanoparticles modified glassy carbon electrode. Electrochemistry Communications, 11(6), 1116-1119.

54. Sherlock, S., \& Dooley, J. (1993) Diseases of the liver and biliary system. Wiley Online Library.

55. Ye, J., Xiong, H., Wang, Q., Zhang, X., Wang, S. (2011) Voltammetric behavior of bilirubin based on [bmim][PF6] as the supporting electrolyte in organic solvent and its analytical application. Am. J. Biomed. Sci, 3(3), 191-198.

56. Wang, L., Wei, L., Chen, Y., Jiang, R. (2010) Specific and reversible immobilization of NADH oxidase on functionalized carbon nanotubes. Journal of Biotechnology, 150(1), $57-63$.

57. Campàs, M., Prieto-Simón, B., Marty, J.L. (2009) A review of the use of genetically engineered enzymes in electrochemical biosensors. Seminars in cell \& developmental biology, 20, 3-9.

58. Taurino, I., Reiss, R., Richter, M., Fairhead, M., ThönyMeyer, L., De Micheli, G., Carrara, S. (2012) Comparative study of three lactate oxidases from Aerococcus viridans for biosensing applications. In preparation. 